\title{
ESTADO TRÓFICO DE UN LAGO TROPICAL DE ALTA MONTAÑA: CASO LAGUNA DE LA COCHA
}

\section{A TROPHIC STATE OF TROPICAL LAKE HIGH MOUNTAIN: CASE OF LAGUNA DE LA COCHA}

\author{
Mery Liliana López Martínez', Sandra Milena Madroñero Palacios²
}

Fecha de recepción: 3 de marzo de 2015

Fecha de aprobación: 12 de agosto de 2015

Referencia: M. L. López Martínez, S. M. Madroñero Palacios. (2015). Estado trófico de un lago tropical de alta montaña: caso laguna de La Cocha. Ciencia e Ingeniería Neogranadina, 25 (2), pp. 21-42, DOl: http://dx.doi.org/10.18359/rcin.1430

\section{RESUMEN}

Este artículo presenta los resultados de una investigación realizada con el fin de determinar el estado trófico de la laguna de La Cocha, cuerpo hídrico importante en Colombia y en el mundo, que hace parte del humedal Ramsar laguna de La Cocha, ecosistema que cumple distintas funciones y alberga una gran biodiversidad que se quiere proteger. Para lograr el propósito planteado, esta investigación se realizó entre enero y septiembre de 2013, determinando el estado trófico mediante los índices de Carlson, el índice desarrollado por la OCDE (Organización para la Cooperación y el Desarrollo Económico) y el índice de Carlson modificado por Toledo. Los resultados permitieron clasificar a la laguna de La Cocha como oligotrófica y ultraoligotrófica, característica típica de lagos de alta montaña con bajos procesos de contaminación de origen aloctóno y autóctono.

Palabras claves: Estado Trófico, Eutroficación, Humedal Ramsar, Mesotrófico, Oligotrófico, Ultraoligotrófico, Laguna de La Cocha.

\section{ABSTRACT}

This article presents the results of a research made in order to determine the trophic status of the Laguna de La Cocha, an important body of water in Colombia and the world, which makes part of the RAMSAR Wetland Laguna de La Cocha, an ecosystem with different functions and the

\footnotetext{
1. Bióloga, Esp., Mg., Docente Asistente, Investigadora Grupo BIOTICS, Universidad Nacional Abierta y a Distancia UNAD, Pasto, Colombia, mery. lopez@unad.edu.co.

2. Bióloga, Mg., M.Sc., Docente, Investigadora del Grupo GIA, Universidad Mariana, Pasto, Colombia, smadronero@umariana.edu.co
} 
home of a big biodiversity that needs protection. To achieve this goal, this research was made between January and September 2013, determining the trophic status using the Carlson's index, the OECD's (Organization for Economic Cooperation and Development) index and the Carlson's index modified by Toledo. The results obtained made it possible to classify the Laguna de La Cocha as oligotrophic and ultraoligotrophic, typical characteristic of high-laying mountain lakes with low pollution processes of allochthonous and autochthonous origin.

Key words: Trophic State, Eutrophication, RAMSAR Wetland, Mesotrophic, Oligotrophic, Ultraoligotrophic, Laguna de La Cocha.

\section{INTRODUCCIÓN}

De la cantidad total de agua disponible en el planeta, el $0,3 \%$ se encuentra en forma de agua dulce líquida superficial $y$, de esta pequeña cantidad, el $90 \%$ se almacena en los lagos; así estos son elementos críticos para la preservación de los ecosistemas y la biodiversidad del planeta. Sin embargo, se encuentran entre los ecosistemas acuáticos más vulnerables y frágiles, ya que son el sumidero de muchas sustancias como: sedimentos, minerales, nutrientes de plantas acuáticas y materiales orgánicos provenientes de su cuenca. La contaminación de los lagos puede surgir por procesos alóctonos y también como consecuencia de las actividades antrópicas que alteran el equilibrio del ecosistema [1].

La laguna de La Cocha (LLC), o lago Guamuez, es un lago tropical de alta montaña que hace parte del humedal de importancia internacional Ramsar laguna de La Cocha. Por su condición de lago tropical de alta montaña, la dinámica de LLC se aparta considerablemente de la que se presenta en los sistemas ubicados en las zonas bajas del trópico y, como lo afirma Gunkel, los lagos tropicales de alta montaña son un tipo especial de ecosistema acuático, de los cuales existe poco conocimiento [2].
Por otra parte, según la Alcaldía Municipal de Pasto [3], la LLC, a pesar de ser un área protegida, presenta un nivel de contaminación medio. Esta es producida por diversos hechos como: el vertido de aguas residuales provenientes de la cabecera corregimental y las veredas circundantes a la LLC, y los hidrocarburos generados por los motores fuera de borda de las lanchas que la transitan.

De igual manera, la producción de carbón vegetal en las zonas aledañas a la LLC es muy alta; la deforestación que esto genera es una de las actividades antrópicas que produce mayor degradación en el bosque primario, secundario, páramos y zonas geológicas de reserva. La actividad carbonera es una gran amenaza, que ha traído como consecuencia erosión de suelos, aumento en la escorrentía de las aguas superficiales y disminución de la biodiversidad [4].

Adicionalmente a la deforestación, en la zona se realiza la siembra de cultivos de papa (Solanum tuberosum L), arveja (Pisum sativum), cebolla junca (Allium fistulosum Linnaeus) y frutas como manzana (Malus domestica), mora (Rubus glaucus), tomate de árbol (cyphomandra betacea) y reinas claudias (Prunas domestica), sin tener en cuenta la aptitud natural del suelo, lo que hace que para mantener los cultivos sea 
necesario utilizar fertilizantes que contienen gran cantidad de nutrientes [5]. De esta manera se incrementa la cantidad de nitrógeno total y fósforo total que llegan al espejo de agua de la LLC, lo que aumenta las concentraciones de los principales nutrientes responsables de los procesos de eutroficación [3].

Cabe resaltar que, a pesar de la importancia que tiene la LLC, no se ha realizado un monitoreo frecuente de sus características morfológicas, hidrológicas, químicas, físicas y biológicas. En el proceso de búsqueda de investigaciones realizadas en la LLC, se determinó que existen estudios dispersos no actualizados. Dada la relevancia de la zona y la necesidad de desarrollar estudios que permitan establecer con certeza el grado de contaminación de la laguna, se realizó esta investigación donde se determinó el estado trófico, el cual se utiliza para clasificar los lagos según la concentración de nutrientes, define el grado de eutroficación y de productividad de un ambiente acuático y sus variaciones en el tiempo [6]. Es un parámetro complejo, definido por Nauman en 1879 como la respuesta biológica de los lagos a la introducción de nutrientes [7-8]. Es un parámetro multidimensional y subjetivo [910]. También es un factor determinante en la calidad del agua, ya que altera la abundancia, diversidad y estructura ecológica de los cuerpos hídricos [2].

Teniendo en cuenta lo mencionado, los cuerpos de agua se clasifican según el estado trófico en: ultraoligotrófico, oligotrófico, mesotrófico, eutrófico e hipertrófico [11]. Esta clasificación se da en un marco específico de condiciones lacustres, y no hay un límite preciso o demarcación fija entre estos. Un lago puede, en cierta época del año, estar en estado oligotrófico y en otra tender a la eutrofia. Lo mismo ocurre en las diferentes zonas que conforman los lagos. Por ejemplo, un lago muy grande puede presentar condiciones oligotróficas en la parte central, mientras en el afluente principal puede estar eutrófica [12].

\section{MATERIALES Y MÉTODOS}

\subsection{UBICACIÓN DEL ÁREA DE ESTUDIO}

La LLC, laguna alto-andina y mayor humedal de los Andes colombianos, está localizada en la vertiente oriental del nudo de Los Pastos, al sur de Colombia en límites con la República de Ecuador (ver figura 1). Hace parte de la cuenca alta del río Guamuez, importante tributario de los ríos Putumayo y San Miguel, afluentes a su vez del río Amazonas [4]. Sus límites la ubican entre las coordenadas: $0^{\circ} 50^{\prime \prime}-1^{\circ} 15^{\prime \prime}$ latitud norte y $77^{\circ} 05^{\prime \prime}-77^{\circ} 20^{\prime \prime}$ longitud oeste [13].

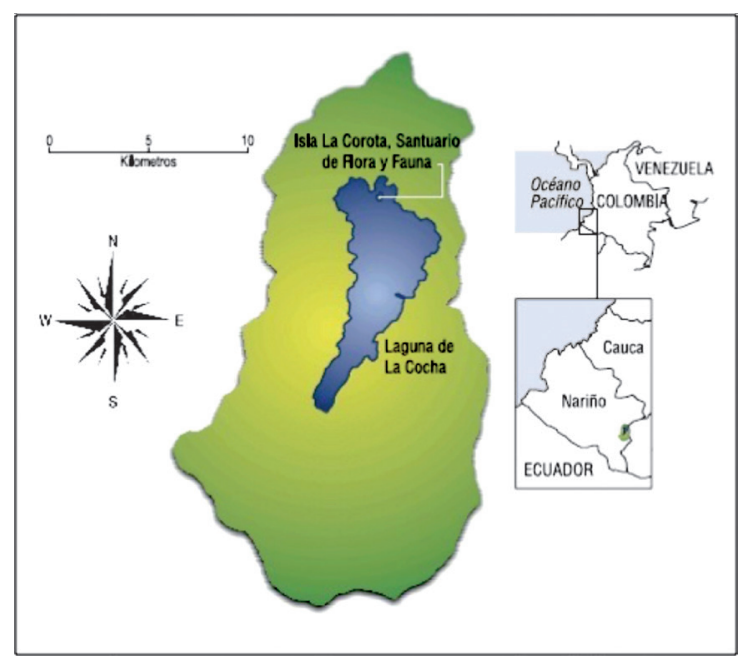

Figura 1. Ubicación geográfica de la laguna de La Cocha. Fuente: Fondo Mundial para la Conservación de la Naturaleza, 2004. 


\subsection{UBICACIÓN DE LOSPUNTOSDE MUESTREO}

Para determinar el estado trófico se calcularon los índices de forma mensual, desde enero hasta junio de 2013 (tabla 1), de tal forma que se pudiera evidenciar la variación temporal del estado trófico de la LLC. Los meses de muestreo abarcaron las épocas de mayor y menor precipitación, los registros de la precipitación media anual en la estación del Encano indican las máximas medias mensuales en los meses de abril, mayo, junio y julio, mientras que los mínimos se presentan en los meses de noviembre, diciembre, enero y febrero [5].

Las mediciones de los diferentes parámetros con los cuales se calcularon los índices, se realizaron en varios puntos representativos de la LLC seleccionados siguiendo la metodología propuesta por Bonilla y Guillot [14]. Se hizo un recorrido en zig-zag de sur a norte, iniciando en la vereda Santa Lucía, y terminando en la entrada nueva del río Encano, con una longitud aproximada de $20 \mathrm{~km}$; se seleccionaron diez puntos de muestreo para tratarr de abarcar la mayor cantidad de ambientes de la LLC (figura 2).

Las coordenadas geográficas de cada punto se obtuvieron con el uso de un receptor de Sistema
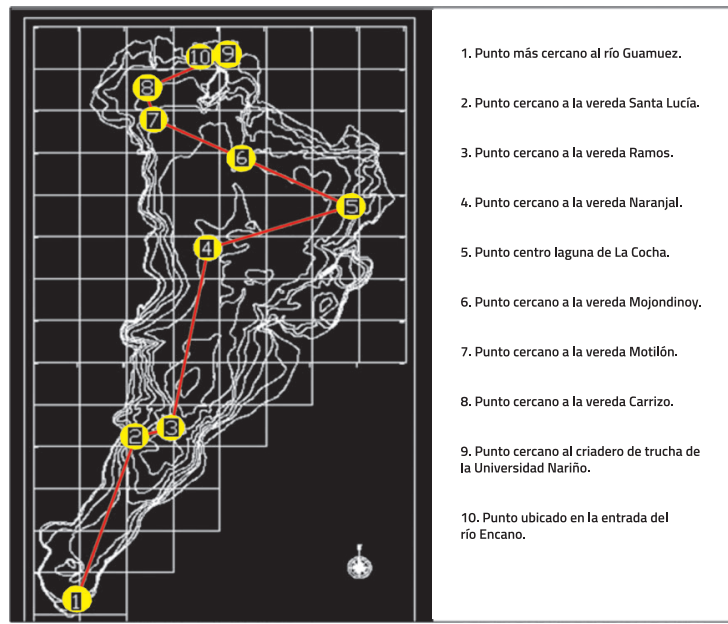

Figura 2. Ubicación de los puntos de muestreo.

de Posicionamiento Global (GPS), marca Garmin, modelo Map 76Csx. Estas coordenadas, la altitud y las principales características de cada punto se indican en la tabla 2.

\subsection{PARÁMETROS FÍSICO-QUÍMICOS CUANTIFICABLES EN LA DETERMINACIÓN DEL ESTADO TRÓFICO}

Para evaluar el estado trófico de un lago se deben tener en cuenta las características morfológicas de la cuenca y las propiedades físicas, químicas

Tabla 1. Fechas de muestreo estado trófico - laguna de La Cocha.

\begin{tabular}{|c|c|c|}
\hline FECHA DE MUESTREO & ÉPOCA & $\begin{array}{c}\text { PRECIPITACIÓN MEDIA MENSUAL } \\
\text { ESTACIÓN ENCANO }\end{array}$ \\
\hline Enero 25 de 2013 & Menor Precipitación & $82,4 \mathrm{~mm}$ \\
\hline Febrero 5 de 2013 & Menor Precipitación & $87,1 \mathrm{~mm}$ \\
\hline Marzo 18 de 2013 & Menor Precipitación & $100,9 \mathrm{~mm}$ \\
\hline Abril 23 de 2013 & Mayor Precipitación & $144,9 \mathrm{~mm}$ \\
\hline Mayo 7 de 2013 & Mayor Precipitación & $155 \mathrm{~mm}$ \\
\hline Junio 12 de 2013 & Mayor Precipitación & $148,3 \mathrm{~mm}$ \\
\hline
\end{tabular}


Tabla 2. Coordenadas puntos de muestreo.

\begin{tabular}{|c|c|c|c|c|}
\hline Punto & $\begin{array}{l}\text { Altura } \\
\text { m.s.n.m. }\end{array}$ & $\begin{array}{l}\text { Latitud } \\
\text { norte }\end{array}$ & Longitud oeste & Características \\
\hline 1 & 2804 & $1^{\circ} 01^{\prime} 00,9^{\prime \prime}$ & $77^{0} 10^{\prime} 36.00^{\prime \prime}$ & $\begin{array}{l}\text { Punto más cercano al río Guamuez, efluente de } \\
\text { la LLC. Se ubica en la vereda Santa Lucía y en su } \\
\text { orilla izquierda se encuentra totora, y vegetación de } \\
\text { bosque muy húmedo montano bajo. }\end{array}$ \\
\hline 2 & 2800 & $1^{\circ} 01^{\prime} 23,1^{\prime \prime}$ & $77^{0} 10^{\prime} 53,4^{\prime \prime}$ & $\begin{array}{l}\text { Existe totora en la orilla y vegetación de bosque muy } \\
\text { húmedo montano bajo, es una zona que no tiene } \\
\text { influencia antrópica. }\end{array}$ \\
\hline 3 & 2796 & $1^{\circ} 03^{\prime} 17,3^{\prime \prime}$ & $77^{\circ} 09^{\prime} 57,6^{\prime \prime}$ & $\begin{array}{c}\text { Este punto se ubicó frente a la vereda Ramos, por } \\
\text { donde corren quebradas que drenan hacia la LLC } \\
\text { y además se desarrollan actividades de ganadería, } \\
\text { de extracción de leche y cultivos de papa (Solanum } \\
\text { tuberosum L). }\end{array}$ \\
\hline 4 & 2796 & $1^{\circ} 03^{\prime} 12,6^{\prime \prime}$ & $77^{\circ} 09^{\prime} 18,4^{\prime \prime}$ & $\begin{array}{l}\text { Punto ubicado cerca a la vereda Naranjal donde } \\
\text { se presentan cultivos minifundistas, y dada la alta } \\
\text { intervención antrópica, se ha intensificado alli la } \\
\text { reforestación para recuperar el bosque natural. }\end{array}$ \\
\hline 5 & 2796 & $1^{\circ} 04^{\prime} 58,7^{\prime \prime}$ & $77^{\circ} 09^{\prime} 03,0^{\prime \prime}$ & $\begin{array}{c}\text { Este punto se ubicó al norte, en el centro de la LLC, } \\
\text { donde históricamente se ha registrado la mayor } \\
\text { profundidad, punto que corresponde a la zona } \\
\text { limnética de la laguna. }\end{array}$ \\
\hline 6 & 2801 & $1^{\circ} 06^{\prime} 13,1^{\prime \prime}$ & $77^{\circ} 07^{\prime} 56,8^{\prime \prime}$ & $\begin{array}{l}\text { Este punto se ubicó cerca a la vereda Mojondinoy, } \\
\text { donde existe una alta producción de carbón vegetal. }\end{array}$ \\
\hline 7 & 2799 & $1^{\circ} 06^{\prime} 52,8^{\prime \prime}$ & $77^{\circ} 08^{\prime} 40,07^{\prime \prime}$ & $\begin{array}{l}\text { Punto ubicado en el flanco occidental, cerca de la } \\
\text { vereda Motilón. Esta vereda está expuesta a riesgo } \\
\text { hidrológico por avenidas torrenciales. }\end{array}$ \\
\hline 8 & 2797 & $1^{\circ} 07^{\prime} 55,5^{\prime \prime}$ & $77^{\circ} 09^{\prime} 59,1^{\prime \prime}$ & $\begin{array}{c}\text { Este punto se ubicó cerca a la vereda El Carrizo, zona } \\
\text { que tiene la más alta concentración de totora. }\end{array}$ \\
\hline 9 & 2799 & $1^{\circ} 08^{\prime} 11,5^{\prime \prime}$ & $77^{\circ} 08^{\prime} 51,2^{\prime \prime}$ & $\begin{array}{c}\text { Se ubicó cerca al criadero de trucha de la Universidad } \\
\text { de Nariño. }\end{array}$ \\
\hline 10 & 2799 & $1^{\circ} 08^{\prime} 09,5^{\prime \prime}$ & $77^{\circ} 09^{\prime} 09,4^{\prime \prime}$ & $\begin{array}{l}\text { Se ubicó en la entrada del río Encano, principal } \\
\text { afluente de la LLC. El sitio es conocido por los } \\
\text { habitantes de la zona como entrada nueva. }\end{array}$ \\
\hline
\end{tabular}

y biológicas del cuerpo de agua. En especial, se deben considerar y medir, la concentración de clorofila "a" (Cl. "a") y la transparencia de la columna de agua. Con los datos obtenidos de estos parámetros posteriormente se aplican las ecuaciones de los índices seleccionados.

\subsubsection{Clorofila "a"}

Para determinar la concentración de clorofila "a" se utilizó el método espectrofotométrico. Se realizó la extracción del pigmento, utilizando como solvente acetona al $90 \%$. Para el cálculo 
de la concentración de la clorofila "a" se utilizó la siguiente ecuación [13]:

Clorofila"a" $\left(\frac{m g}{m^{3}}\right)=\frac{\{26,7 \cdot(665 A d-665 A a) \cdot V 1\}}{V 2 . L c}$

Donde:

665Ad: Lectura de absorbancia a $665 \mathrm{~nm}$ antes de la acidificación.

665Aa: Lectura de absorbancia a 665 nm después de la acidificación.

V1: Volumen de acetona utilizado en la extracción en litros.

V2: Volumen de muestra filtrado en litros.

LC: $\quad$ Longitud de la celda espectrofotométrica en centímetros.

\subsubsection{Transparencia de la columna de agua}

La transparencia se midió con el disco Secchi, que es un dispositivo de 20 a 30 centímetros de diámetro, dividido en cuartos, que se pintan de blanco y negro alternativamente para mejorar el contraste. Se lo introdujo en el agua, dejándolo caer hasta que se perdiera de vista y allí se registró la profundidad. Después se lo sumergió nuevamente, a mayor profundidad que la anterior, hasta que no se lo pudiera observar, para luego subirlo y hacer el registro en el momento en el que fue visible nuevamente. Se promedió la medida de las dos profundidades y ese valor, expresado en metros, es la transparencia medida por el disco Secchi.

\section{4 ÍNDICES DE ESTADO TRÓFICO}

El estado trófico de un cuerpo de agua permite evaluar su calidad a través de indicadores matemáticos, llamados índices de estado trófico [16]. Hay gran cantidad de estos para establecer el estado trófico de un sistema léntico. A continuación se describen los seleccionados para el desarrollo de esta investigación.

\subsection{1 Îndice propuesto por la Organización para la Cooperación y el Desarrollo Económico (OCDE)}

Luego de un estudio que durante 5 años abarcó 200 ambientes en 22 países de Europa occidental, Estados Unidos, Japón y Australia, el Comité de Eutroficación de la Organización para la Cooperación y el Desarrollo Económico (OCDE) en 1982, propuso una clasificación del grado de eutrofia de lagos y embalses. La OCDE se propuso definir un lenguaje común para los parámetrosa medir, buscando una simplificación de estos, pero a la vez seleccionando aquellos con significado biológico adecuado, para un análisis estadístico reproducible [17].

Esta clasificación proporciona un esquema cuantitativo probabilístico para las diferentes categorías de estado trófico a partir del análisis de nutrientes (concentración de fósforo), de la capacidad de generar biomasa (clorofila "a") y de la disponibilidad de espectro fotosintético en la vertical de la columna de agua (transparencia de la columna de agua). Los datos de fósforo total, transparencia de la columna de agua y clorofila "a", se relacionan con el estado trófico asignado de acuerdo con las percepciones cualitativas, tal como lo informaron Vollenweider y Kerekes en 1981 [18]. En este índice los valores varían en una escala de cero a cien [19] y los criterios de aplicación se presentan en la tabla 3:

Una vez realizadas las mediciones con el disco Secchi y determinada la concentración de clorofila "a", se elaboró una base de datos en Excel, para comparar los valores obtenidos 
Tabla 3. Criterios de aplicación del Índice OCDE.

\begin{tabular}{|c|c|c|c|}
\hline Categoría trófica & $\begin{array}{c}\text { Clorofila "a" } \\
(\boldsymbol{\mu g} / \mathbf{L})\end{array}$ & $\begin{array}{c}\text { Profundidad Secchi } \\
\text { (metros) }\end{array}$ & $\begin{array}{c}\text { Fósforo } \\
\text { ( } \mathbf{\mu g} / \mathbf{L})\end{array}$ \\
\hline Ultraoligotrófico & $<1$ & $>12$ & $<4$ \\
\hline Oligotrófico & $1-2,5$ & 6 a 12 & 4 a 10 \\
\hline Mesotrófico & 2,5 a 7,9 & 3 a 6 & 10 a 35 \\
\hline Eutrófico & 8 a 25 & 1,5 a 3 & 35 a 100 \\
\hline Hipertrófico & $>25$ & $<1,5$ & $>100$ \\
\hline
\end{tabular}

con los valores de referencia de la OCDE, y así establecer la categoría trófica, cada mes, en todos los puntos donde se tomaron muestras.

\subsection{2 Îndice de Carlson o índice de estado trófico (IET)}

Propuesto en 1977 [20], ha sido uno de los primeros índices propuestos para sistemas lacustres; está basado en la utilización del Disco de Secchi para la medición de la transparencia del agua a través de la columna de agua del lago. Esta transparencia determina el nivel de refracción de la luz a través de la turbidez y el color que presenta el volumen de agua, por efecto de descargas de sólidos (suspendidos, volátiles o sedimentables) o por la formación de sistemas coloidales o soluciones complejas [21]. Después de publicado el trabajo de Carlson, en el año de 1981, Alzaki propuso una modificación al Índice de Carlson a través de la inclusión de coeficientes específicos para cada elemento que integra la composición del estado trófico, se cuantifica la biomasa a partir de la medición de la clorofila tipo "a" [22].

El índice de estado trófico de Carlson se calcula con las siguientes ecuaciones:

$$
I E T D S=10\left(6-\frac{\ln (D S)}{\ln (2)}\right)
$$

$$
\text { IETCl. "a" }=10\left(6-\frac{2,04-0,68 \ln \left(C l . " a^{\prime \prime}\right)}{\ln (2)}\right)
$$

$$
\text { IET Promedio }=\frac{\text { IETDS }+I E T C l . " a "}{2}
$$

Donde:

IETDS: Índice de estado trófico de Carlson disco Secchi.

IETCI. "a": Índice de estado trófico de Carlson clorofila "a".

IET Promedio: Índice de estado trófico de Carlson promedio.

Los valores adimensionales obtenidos al aplicar las ecuaciones (2), (3) y (4) varían en una escala de cero a cien [21 - 23] y el criterio de aplicación se resume en la Tabla 4:

Tabla 4. Criterios de aplicación del IET.

\begin{tabular}{|c|c|}
\hline Valor İndice & $\begin{array}{c}\text { Categoría Trófica } \\
<30\end{array}$ \\
\hline $30-44$ & Ultraoligotrófico \\
\hline $44-54$ & Oligotrófico \\
\hline $54-74$ & Mesotrófico \\
\hline$>74$ & Eutrófico \\
\hline
\end{tabular}




\subsection{3. Índice de estado trófico de Carlson modificado por Toledo (IETM).}

Toledo, en 1985 [10], propuso modificaciones en la formulación matemática del IET con el fin de adaptarlo a las condiciones climáticas de los ambientes tropicales, modificación aplicada en un estudio realizado en un reservorio ubicado en la población de Barra Bonita en Brasil [8].

El índice de estado trófico de Carlson modificado por Toledo, se calcula con las siguientes ecuaciones:

$$
\begin{gathered}
\text { IETMDS }=10\left(6-\frac{0,64+\ln (D S)}{\ln (2)}\right) \\
\text { IETMCl. "a" }=10\left(6-\frac{2,04-0,695 \ln \left(C l . " a^{\prime \prime}\right)}{\ln (2)}\right) \\
\text { IETM Promedio }=\frac{\text { IETMDS+IETMCl." a" }}{2}
\end{gathered}
$$

Donde:

IETMDS: Índice de estado trófico de Carlson modificado por Toledo Disco Secchi

IETMCI. "a": Índice de estado trófico de Carlson modificado por Toledo Clorofila "a"

IETM promedio: Índice de estado trófico de Carlson promedio modificado por Toledo

Los valores obtenidos al aplicar las ecuaciones (5), (6) y (7), varían en una escala de cero a cien y los criterios de aplicación se resumen en la tabla 4, citada anteriormente.

Para el análisis estadístico del IET y el IETM se utilizó la media aritmética $(\bar{X})$ como medida de tendencia central, la desviación estándar (DE) como medida de dispersión absoluta y el coeficiente de variación de Pearson (CV, \%) como medida de dispersión relativa.

\section{RESULTADOS Y ANÁLISIS}

Para iniciar es importante explicar la ausencia de datos en el muestreo del mes de abril de 2013 en el punto 8. No fue posible la toma de muestras porque las condiciones climáticas del lugar impidieron acceder al punto.

\subsection{TRANSPARENCIA DE LA COLUMNA DE AGUA.}

Los resultados obtenidos de la transparencia de la columna de agua se muestran en la tabla 5.

Los valores más altos de transparencia de la columna de agua se registraron en el mes de febrero, pero ni el máximo valor encontrado en este estudio, que fue de 8 metros, alcanza el valor de transparencia de 13 metros informado por Roldán y Ramírez [24] para la LLC; esta diferencia puede darse por la fecha y el sitio de muestreo en el cual se haya realizado el estudio, condiciones que no se encuentran especificadas en el libro.

Pero López et al. [25] informaron valores de transparencia entre 3,5 y 4,6 metros, valores similares a los encontrados en esta investigación. Al igual que este estudio, se hallaron los menores valores de transparencia en cercanías a la vereda El Puerto, lecturas que pueden explicarse por las condiciones particulares de la zona donde hay carencia de alcantarillado, de sistema de tratamiento de aguas servidas y eliminación de excretas, las cuales se vierten directamente en el cauce del río El Encano. 
Tabla 5. Valores de la transparencia de la columna de agua en la laguna de La Cocha.

\begin{tabular}{|c|c|c|c|c|c|c|c|}
\hline \multirow{2}{*}{ Mes } & \multicolumn{7}{|c|}{ Profundidad disco Secchi en metros } \\
\cline { 2 - 8 } & Enero & Febrero & Marzo & Abril & Mayo & Junio & Promedio \\
\hline $\mathbf{1}$ & 4,9 & 4 & 5,1 & 4,7 & 3,8 & 3,7 & 4,37 \\
\hline $\mathbf{2}$ & 4,4 & 2,7 & 5,6 & 4,7 & 4,6 & 3,4 & 4,23 \\
\hline $\mathbf{3}$ & 5,3 & 6,2 & 5,8 & 4 & 4,5 & 3,4 & 4,87 \\
\hline $\mathbf{4}$ & 5 & 7 & 5,5 & 4,6 & 4,6 & 2,9 & 4,93 \\
\hline $\mathbf{5}$ & 5,5 & 6,4 & 4,8 & 4,6 & 3,5 & 3,4 & 4,70 \\
\hline $\mathbf{6}$ & 5,3 & 7,4 & 5,1 & 3,5 & 3,6 & 3,1 & 4,67 \\
\hline $\mathbf{7}$ & 5,4 & 8 & 4,5 & 3,5 & 3,3 & 3,2 & 4,65 \\
\hline $\mathbf{8}$ & 4,6 & 7 & 5,1 & 5.0 & 3,4 & 3,2 & 4,66 \\
\hline $\mathbf{9}$ & 4,4 & 6,5 & 5 & 2,4 & 3,8 & 2,4 & 4,08 \\
\hline $\mathbf{1 0}$ & 4,1 & 4 & 4,3 & 2,2 & 3,2 & 2,9 & 3,45 \\
\hline Promedio & 4,89 & 5,92 & 5,08 & 3,8 & 3,83 & 3,16 & \\
\hline
\end{tabular}

También en el punto 9 de esta investigación, se encontraron valores bajos de transparencia, que oscilaron entre los 2,4 y 6,5 metros. Este punto se ubicó cerca al criadero de trucha de la Universidad de Nariño, y los resultados obtenidos en el punto concuerdan con lo encontrado por López et al. [25]. La transparencia es menor en el centro y en los alrededores de las jaulas, y aumenta a medida que se toman lecturas en zonas alejadas de las jaulas flotantes.

Los demás puntos de esta investigación, identificados como 1, 2, 3, 4, 5, 6, 7 y 8, mostraron un comportamiento similar que se asemeja por completo a lo informado en el Plan de Manejo Ambiental del Humedal Ramsar laguna de La Cocha [5], cuya transparencia promedio se midió en 4,5 metros.
En lagos de alta montaña, como la LLC, se encontraron informes de transparencia que varían con las características particulares de cada lago. Por ejemplo, Casallas y Gunkel [26] informaron para el lago San Pablo de Ecuador, valores de transparencia en 2,1 y 4,2 metros, con un valor medio de 3 metros, valores más bajos si se comparan con los de la LLC. Esta diferencia obedece a la alta intervención antrópica y al fuerte proceso de eutroficación que se da en el lago San Pablo.

De igual manera Iltis, Carmouze y Lemoalle [27] informaron valores de transparencia del lago Titicaca en Bolivia. Los valores promedio para la zona conocida como el lago Menor están en el rango de 4,7 a 5,4 metros, y para el lago Mayor entre 4,5 a 10,5 metros, diferencias explicables por la época del año y las actividades 
particulares presentes en cada zona, donde los menores valores de transparencia se dan en zonas de fuerte influencia antrópica.

Finalmente, es importanteaclarar que una buena interpretación de los valores de transparencia depende de las condiciones particulares de cada zona y de la presencia o ausencia de materiales suspendidos, ya que, como afirma Carlson [20], la transparencia puede presentar errores cuando los lagos tienen altas concentraciones de materia en suspensión o de color.

\subsection{CLOROFILA "a".}

Los valores de concentración de clorofila "a" obtenidos después de aplicar la ecuación (1), se presentan en la tabla 6 .

No hay una diferencia en la concentración de clorofila "a" entre los meses en que se realizó este estudio. Se encontró una diferencia entre los puntos, donde todos presentan un comportamiento similar, a excepción del punto 9, donde se midieron los valores más altos de clorofila "a". Este punto está ubicado cerca al criadero de trucha de la Universidad de Nariño. Las truchas criadas en los estanques son alimentadas con concentrados, que presentan gran cantidad de fósforo y nitrógeno. Todas las partículas de alimento no son ingeridas por los peces, algunas quedan en el agua, haciendo que aumente el contenido de nutrientes, lo que trae como consecuencia un mayor crecimiento del fitoplancton y una mayor concentración de clorofila "a" [28].

Situación similar a la explicada, fue encontrada en el estudio de López et al. [25], realizado también en la LLC, en el cual encontraron concentraciones de clorofila "a" entre 2,47 y 7,3 $\mu \mathrm{g} / \mathrm{L}$. Estos valores presentan diferencias

Tabla 6. Valores de concentración de clorofila "a" ( $\mu \mathrm{g} / \mathrm{L})$ en la Laguna de La Cocha.

\begin{tabular}{|c|c|c|c|c|c|c|c|}
\hline MES & Enero & Febrero & Marzo & Abril & Mayo & Junio & Promedio \\
\hline $\mathbf{1}$ & 1,07 & 1,94 & 1,18 & 1,14 & 2,08 & 1,07 & 1,41 \\
\hline $\mathbf{2}$ & 0,53 & 1,25 & 1,26 & 1,19 & 1,28 & 0,98 & 1,08 \\
\hline $\mathbf{3}$ & 1,07 & 1,1 & 1,94 & 1,86 & 1,07 & 1,46 & 1,42 \\
\hline $\mathbf{4}$ & 1,16 & 1,17 & 1,21 & 1,37 & 1,3 & 1,11 & 1,22 \\
\hline $\mathbf{5}$ & 1,19 & 1,38 & 1,51 & 1,23 & 1,25 & 1,25 & 1,30 \\
\hline $\mathbf{6}$ & 1,21 & 1,48 & 1,56 & 1,07 & 1,59 & 1,38 & 1,38 \\
\hline $\mathbf{7}$ & 1,9 & 1,82 & 1,18 & 1,21 & 1,59 & 1,79 & 1,58 \\
\hline $\mathbf{8}$ & 1,01 & 1,62 & 1,94 & 5.0 & 1,62 & 2 & 1,64 \\
\hline $\mathbf{9}$ & 2,31 & 2,58 & 2,26 & 2,34 & 2,52 & 2,17 & 2,36 \\
\hline $\mathbf{1 0}$ & 1,47 & 2,48 & 1,44 & 1,39 & 2,36 & 1,85 & 1,83 \\
\hline Promedio & 1,29 & 1,68 & 1,55 & 1,42 & 1,67 & 1,51 & \\
\hline
\end{tabular}


con los valores de esta investigación, porque en dicho estudio todas las muestras de agua se tomaron asociadas a criaderos de truchas, los valores más altos se hallaron siempre en la parte central de todos los criaderos y los valores más bajos se encontraron a una distancia de 20 metros alejados de las jaulas. Sin embargo, los valores informados para los sitios alejados de las jaulas de las truchas son similares a los encontrados en esta investigación.

Asimismo, en el Plan de Manejo Ambiental del Humedal Ramsar laguna de La Cocha [5], se informaron altos valores de clorofila, que oscilan entre 7,44 y 9,3 $\mu \mathrm{g} / \mathrm{L}$, y evidenciarían un proceso de eutroficación. Al profundizar en estos resultados, en el documento no se encontró un análisis detallado de estos, ni denominación de los puntos donde se obtuvieron los datos, ni especificación del método de extracción del pigmento. Por las razones expuestas, estos valores no permiten comparación alguna con los encontrados en esta investigación.

Luego, con el fin de evidenciar las consecuencias de un alto o bajo nivel en la concentración de clorofila "a", se consultaron datos en otros ecosistemas lénticos ubicados en Suramérica, encontrando que en el estudio de Díaz et al. [29], realizado en los lagos de los Andes argentinos, se informaron concentraciones bajas de clorofila "a" con valores menores a $3 \mu \mathrm{g} / \mathrm{L}$; en su análisis afirman que las bajas concentraciones de clorofila se pueden deber a una baja concentración de nutrientes, característica de los lagos ubicados en la cordillera de los Andes, como sería el caso de la LLC.

De igual manera, en el lago San Pablo, ubicado en Ecuador, Gunkel [30] informó para la concentración de clorofila "a" valores promedio de 9,36 $\mu \mathrm{g} / \mathrm{L}$, valores que se consideraron altos y evidenciaron un proceso de eutroficación en ese ecosistema, postulado que se validó con las demás variables que se analizaron en ese estudio. También Aubriot et al. [31] informaron que en la laguna de Rocha, Humedal Ramsar ubicado en Uruguay, la concentración de clorofila "a" osciló entré 9,7 $\pm 7,1 \mu \mathrm{g} / \mathrm{L}$ y $6,4 \pm 4,6 \mu \mathrm{g} / \mathrm{L}$, y basados en esos valores concluyeron que la laguna de Rocha es altamente vulnerable y presenta evidencias de procesos de eutroficación acelerados por las actividades antrópicas presentes en esa zona.

Finalmente, para realizar la clasificación del estado trófico, es importante tener en cuenta lo afirmado por Coelho et al. [32] y Condé [8] quienes concluyeron después de sus investigaciones en Portugal y São Paulo (Brasil), que el mejor referente para dar la clasificación es la biomasa, porque sus datos son más confiables y tienen menos errores que los datos de transparencia.

\section{3 ÍNDICE OCDE.}

Con los resultados obtenidos de la concentración de clorofila "a" y la transparencia de la columna de agua, se procedió a determinar el estado trófico aplicando el índice OCDE. Los resultados obtenidos se presentan en la tabla 7, por cada punto de muestreo, por cada mes y por cada parámetro medido.

Los resultados de este índice, al aplicarlo a la concentración de clorofila "a", indican que todos los puntos durante todos los meses de muestreoseclasificaroncomoultraoligotróficos y oligotróficos. De igual manera, aplicado el índice OCDE a la transparencia de la columna de agua, se encontró que los resultados 
Tabla 7. Estado trófico según el índice OCDE en la laguna de La Cocha.

\begin{tabular}{|c|c|c|c|c|c|c|c|}
\hline \multirow{2}{*}{ Punto } & \multirow{2}{*}{ Parámetros } & \multicolumn{6}{|c|}{ Mes de muestreo } \\
\hline & & Ene. & Feb. & Mar. & Abr. & May. & Jun. \\
\hline \multirow{2}{*}{1} & Transparencia & Meso. & Meso. & Meso. & Meso. & Meso. & Meso. \\
\hline & Clorofila "a" & Oligo. & Oligo. & Oligo. & Oligo. & Oligo. & Oligo. \\
\hline \multirow{2}{*}{2} & Transparencia & Meso. & Eu. & Meso. & Meso. & Meso. & Meso. \\
\hline & Clorofila "a" & Ultra. & Oligo. & Oligo. & Oligo. & Oligo. & Ultra. \\
\hline \multirow{2}{*}{3} & Transparencia & Meso. & Oligo. & Meso. & Meso. & Meso. & Meso. \\
\hline & Clorofila "a" & Oligo. & Oligo. & Oligo. & Oligo. & Oligo. & Oligo. \\
\hline \multirow{2}{*}{4} & Transparencia & Meso. & Oligo. & Meso. & Meso. & Meso. & Eu. \\
\hline & Clorofila "a" & Oligo. & Oligo. & Oligo. & Oligo. & Oligo. & Oligo. \\
\hline \multirow{2}{*}{5} & Transparencia & Meso. & Oligo. & Meso. & Meso. & Meso. & Meso. \\
\hline & Clorofila "a" & Oligo. & Oligo. & Oligo. & Oligo. & Oligo. & Oligo. \\
\hline \multirow{2}{*}{6} & Transparencia & Meso. & Oligo. & Meso. & Meso. & Meso. & Meso. \\
\hline & Clorofila "a" & Oligo. & Oligo. & Oligo. & Oligo. & Oligo. & Oligo. \\
\hline \multirow{2}{*}{7} & Transparencia & Meso. & Oligo. & Meso. & Meso. & Meso. & Meso. \\
\hline & Clorofila "a" & Oligo. & Oligo. & Oligo. & Oligo. & Oligo. & Oligo. \\
\hline \multirow{2}{*}{8} & Transparencia & Meso. & Oligo. & Meso. & S.D & Meso. & Meso. \\
\hline & Clorofila "a" & Oligo. & Oligo. & Oligo. & S.D & Oligo. & Oligo. \\
\hline \multirow{2}{*}{9} & Transparencia & Meso. & Oligo. & Meso. & Eu. & Meso. & Eu. \\
\hline & Clorofila "a" & Oligo. & Oligo. & Oligo. & Oligo. & Oligo. & Oligo. \\
\hline \multirow{2}{*}{10} & Transparencia & Meso. & Meso. & Meso. & Eu. & Meso. & Eu. \\
\hline & Clorofila "a" & Oligo. & Oligo. & Oligo. & Oligo. & Oligo. & Oligo. \\
\hline
\end{tabular}

Ultra. = Ultraoligotrófico; Oligo. = Oligotrófico; Meso. = Mesotrófico; Eu. = Eutrófico. S.D. = Sin Datos.

variaron entre las categorías oligotrófica a eutrófica. El $11,86 \%$ de los datos se ubicaron en la categoría oligotrófica, el $79,66 \%$ en la categoría mesotrófica y el 8,47\% en la categoría eutrófica.

La interpretación del índice OCDE es algo compleja, porque se analiza cada parámetro por separado sin que pueda haber integración alguna. Si bien esta condición se convierte en una limitante, refleja claramente que el estado trófico es de carácter multidimensional, porque, como lo afirman Janus y Vollenweider, es imposible establecer una limitación estricta entre las distintas categorías tróficas [22]. 
Como para la LLC no se encontraron determinaciones del estado trófico según el índice OCDE, no se puede hacer comparación con estudios anteriores, pero sí con otros lagos tropicales de alta montaña. Según el índice OCDE la LLC es oligotrófica para la concentración de clorofila "a" y oligotrófica, mesotrófica y eutrófica según los valores de transparencia en los diferentes puntos, situación que refleja lo que se dijo anteriormente sobre las condiciones particulares de cada zona y de la presencia o ausencia de materiales suspendidos. Este resultado concuerda con el informado para el lago San Pablo [26], al cual se le aplicó el índice OCDE y se lo clasificó como lago oligotrófico según los valores de clorofila "a" y eutrófico según los valores de la transparencia de la columna de agua. Se observa claramente que se obtienen resultados dicotómicos, que como lo afirman Ludovisi y Poletti [19], pueden causar confusión si no se interpretan de acuerdo con las características particulares de cada zona.

Procediendo a analizar las características de cada punto, se observa que no hay variación en cuanto a la concentración de clorofila "a" en todos los puntos de muestreo, pero existe variación en la transparencia, porque los resultados de los puntos 9 y 10 se alejan de los resultados de los otros puntos. Puede ser que el punto 9, ubicado cerca de un criadero de trucha, presente una menor transparencia de la columna de agua, por la suspensión de partículas provenientes del concentrado utilizado para alimentar a los peces, situación informada en el estudio que realizó López et al. en la LLC [25].

En el punto 10, cercano a la desembocadura del río Encano, hay presencia de gran cantidad de material arrastrado por el río, además de actividades antrópicas como: tránsito de lanchas con motores fuera de borda, presencia de residuos sólidos y descargas de aguas residuales domésticas, que pueden influir en los resultados de transparencia en la columna de agua. La misma situación se encontró en el lago Titicaca, que se estudió en 4 estaciones de muestreo, encontrando condiciones eutróficas en la Bahía Cohana donde se practica cría intensiva de ganado, y en la localidad de Tiquina, que posee el criadero de truchas más grande de la parte boliviana del lago [33].

Los otros puntos de esta investigación, identificados como 1, 2, 3, 4, 5, 6, 7 y 8, mostraron un comportamiento similar. Según la transparencia, todos fueron clasificados como mesotróficos, y según la clorofila "a" todos fueron clasificados como oligotróficos, evidenciando que no existe una variación espacial, ni temporal para el estado trófico de la LLC.

Finalmente, es importante aclarar que no existen muchos estudios en lagos tropicales de alta montaña donde se aplique el índice OCDE. Generalmente este índice se utiliza para ecosistemas de zonas templadas [34], y para definir el estado trófico de ecosistemas lénticos tropicales se han desarrollado otro tipo de metodologías, especialmente para lagos cálidos tropicales [18], pero para lagos tropicales de alta montaña no se han propuesto otras metodologías debido a la complejidad de esos ecosistemas.

\section{4 ÍNDICE DE ESTADO TRÓFICO DE CARLSON.}

Este índice permitió hacer un análisis cualitativo y cuantitativo de los parámetros. Los resultados que se obtuvieron al aplicar las ecuaciones, se interpretaron según los criterios presentados en la tabla 4. El valor adimensional obtenido 
en cada punto permitió clasificarlo en una categoría trófica. Los resultados encontrados para todos los puntos se resumen en la tabla 8.

El IET para la clorofila "a" indicó que no existe variación espacial, ni temporal para la cantidad de biomasa en la LLC, lo que permite su clasificación en su estado trófico como oligotrófica, condición informada también para lagos de alta montaña por Quirós quien en estudios en lagos de los Andes Argentinos, afirma que ésta situación se puede presentar

Tabla 8. Estado trófico según el IET en la Laguna de La Cocha.

\begin{tabular}{|c|c|c|c|c|c|c|c|}
\hline \multirow{2}{*}{ PUNTO } & \multirow{2}{*}{ ESTADO TRÓFICO } & \multicolumn{6}{|c|}{ MES DE MUESTREO } \\
\hline & & ENE. & FEB. & MAR. & ABR. & MAY. & JUN. \\
\hline \multirow[t]{2}{*}{1} & IETDS & Oligo. & Oligo. & Oligo. & Oligo. & Oligo. & Oligo. \\
\hline & IET CI."a" & Oligo. & Oligo. & Oligo. & Oligo. & Oligo. & Oligo. \\
\hline \multirow[t]{2}{*}{2} & IETDS & Oligo. & Meso. & Oligo. & Oligo. & Oligo. & Oligo. \\
\hline & IET CI."a" & Ultra. & Oligo. & Oligo. & Oligo. & Oligo. & Oligo. \\
\hline \multirow[t]{2}{*}{3} & IETDS & Oligo. & Oligo. & Oligo. & Oligo. & Oligo. & Oligo. \\
\hline & IET Cl."a" & Oligo. & Oligo. & Oligo. & Oligo. & Oligo. & Oligo. \\
\hline \multirow[t]{2}{*}{4} & IETDS & Oligo. & Oligo. & Oligo. & Oligo. & Oligo. & Meso. \\
\hline & IET CI."a" & Oligo. & Oligo. & Oligo. & Oligo. & Oligo. & Oligo. \\
\hline \multirow[t]{2}{*}{5} & IET DS & Oligo. & Oligo. & Oligo. & Oligo. & Oligo. & Oligo. \\
\hline & IET CI."a" & Oligo. & Oligo. & Oligo. & Oligo. & Oligo. & Oligo. \\
\hline \multirow[t]{2}{*}{6} & IET DS & Oligo. & Oligo. & Oligo. & Oligo. & Oligo. & Oligo. \\
\hline & IET CI."a" & Oligo. & Oligo. & Oligo. & Oligo. & Oligo. & Oligo. \\
\hline \multirow[t]{2}{*}{7} & IET DS & Oligo. & Oligo. & Oligo. & Oligo. & Oligo. & Oligo. \\
\hline & IET CI."a" & Oligo. & Oligo. & Oligo. & Oligo. & Oligo. & Oligo. \\
\hline \multirow[t]{2}{*}{8} & IET DS & Oligo. & Oligo. & Oligo. & S.D & Oligo. & Oligo. \\
\hline & IET Cl."a" & Oligo. & Oligo. & Oligo. & S.D & Oligo. & Oligo. \\
\hline \multirow[t]{2}{*}{9} & IET DS & Oligo. & Oligo. & Oligo. & Meso. & Oligo. & Meso. \\
\hline & IET Cl."a" & Oligo. & Oligo. & Oligo. & Oligo. & Oligo. & Oligo. \\
\hline \multirow[t]{2}{*}{10} & IET DS & Oligo. & Oligo. & Oligo. & Meso. & Oligo. & Meso. \\
\hline & IET CI."a" & Oligo. & Oligo. & Oligo. & Oligo. & Oligo. & Oligo. \\
\hline
\end{tabular}

Ultra. = Ultraoligotrófico; Oligo. = oligotrófico; Meso. = mesotrófico y Eu. = eutrófico 
en lagos donde hay deficiencia de nutrientes y además la actividad antrópica no ha logrado afectar los ecosistemas [35].

El IET aplicado a la transparencia de la columna de agua clasificó a LLC como oligotrófica, a excepción de los puntos 9 y 10 en los cuales se clasificó como mesotrófica en los meses de abril y junio. Estos resultados coinciden con el estudio de López et al. [25] quienes clasificaron la LLC según la transparencia de agua, como oligotrófica y mesotrófica con valores más bajos, en sitios cercanos a los criaderos de trucha. El estudio de López et al. [25], no analizó la zona limnética de la LLC, razón por la cual no puede compararse con los resultados obtenidos en los otros puntos de muestreo de esta investigación.

En esta investigación, el resultado del IET promedio es el mismo informado en los estudios de López et al. [25] y del Ministerio del Medio Ambiente - Corponariño [5], que clasificaron a la LLC como oligotrófica utilizando el IET.

De nuevo se evidencia el poco conocimiento acerca de ecosistemas tropicales de alta montaña, pues no se encontraron muchos estudios donde se aplique este índice. Los estudios encontrados para ecosistemas donde se aplica el IET, corresponden a ecosistemas de zonas templadas y de zonas tropicales cálidas. Pese a esta situación, se encontró que los resultados de la LLC concuerdan con los de otros estudios donde se aplicó el IET. Betancourt et al. [37] en el embalse de Abreus en Cuba, y Calazans et al. [16] en tres lagos del río Grande del Norte (Brasil), concluyeron que los cuerpos de agua tienden a la eutroficación cuando reciben descargas de aguas residuales, condición que aumenta la concentración de nutrientes en el agua $y$, por ende, aumenta la biomasa. De igual forma Li et al. [38] encontraron que en el
Parque Nacional Xixi de China los procesos de eutroficación son más evidentes en cuerpos de agua cercanos a ciudades o a cultivos, pues reciben una gran carga de nutrientes que aumentan la cantidad de biomasa.

Cuantitativamente, se aplicó el IET promedio que pretende conservar la simplicidad de la unidimensionalidad que, como lo afirman Velásquez et al. [36] es un problema no fácil de resolver, ya que algunos lagos pueden ser considerados eutróficos por un criterio y oligotróficos por otro, tal y como se presentó en este estudio. Con el IET promedio se realizó el análisis estadístico cuyos resultados se pueden observar en la tabla 9.

Los valores del IET promedio más altos se presentaron en los sitios donde hay mayor intervención antrópica (puntos 9 y 10) donde hay presencia de un criadero de trucha, llegada de un afluente y cercanía a una población de la cual se reciben aguas residuales domésticas y residuos sólidos, situación que ya se explicó anteriormente en el análisis del índice OCDE.

El coeficiente de variación para cada punto de muestreo y para cada mes de muestreo, arrojó valores inferiores a $10 \%$. Lo que permite afirmar que, según las orientaciones de Canavos [39], los datos fueron homogéneos tanto en los meses de mayor como de menor precipitación. En el punto 5, ubicado en el centro de la LLC, se encontró el menor valor del coeficiente de variación con un $4,58 \%$, y el valor más alto fue de $7,04 \%$ en el punto 8 , ubicado en la vereda Carrizo. Teniendo en cuenta los meses de muestreo, en marzo se encontró el valor más bajo del coeficiente de variación, 3,27 \% y en febrero se encontró el valor más alto con un $8,20 \%$.

Por otra parte, el análisis estadístico para el IET promedio indicó que no se presentó variación 
Tabla 9. Valores promedios, X , D.E y C.V para el IET en la Laguna de La Cocha.

\begin{tabular}{|c|c|c|c|c|c|c|c|c|c|}
\hline \multirow{2}{*}{ Punto } & \multicolumn{6}{|c|}{ Valores promedios IET. } & \multirow{2}{*}{$\mathrm{X}$} & \multirow{2}{*}{ D.E } & \multirow{2}{*}{$\begin{array}{c}\text { C.V } \\
\%\end{array}$} \\
\hline & Ene. & Feb. & Mar. & Abr. & May. & Jun. & & & \\
\hline 1 & 34,15 & 38,62 & 34,35 & 34,75 & 39,24 & 36,26 & 36,20 & 2,04 & 5,62 \\
\hline 2 & 31,46 & 39,23 & 34,00 & 34,96 & 35,49 & 36,37 & 35,25 & 2,35 & 6,67 \\
\hline 3 & 33,65 & 32,59 & 35,85 & 38,32 & 34,77 & 38,30 & 35,57 & 2,17 & 6,10 \\
\hline 4 & 34,39 & 32,01 & 33,91 & 35,82 & 35,56 & 38,25 & 34,97 & 1,92 & 5,48 \\
\hline 5 & 33,72 & 33,48 & 36,07 & 35,27 & 37,45 & 37,64 & 35,58 & 1,63 & 4,58 \\
\hline 6 & 34,27 & 32,81 & 35,72 & 36,70 & 38,21 & 38,82 & 36,05 & 2,10 & 5,83 \\
\hline 7 & 36,26 & 33,21 & 35,32 & 37,18 & 38,95 & 39,75 & 36,77 & 2,19 & 5,96 \\
\hline 8 & 34,32 & 33,61 & 36,78 & S.D & 38,84 & 40,42 & 36,77 & 2,59 & 7,04 \\
\hline 9 & 38,69 & 36,50 & 37,53 & 43,30 & 40,18 & 43,24 & 39,82 & 2,63 & 6,59 \\
\hline 10 & 37,01 & 39,84 & 36,63 & 41,37 & 41,11 & 40,61 & 39,37 & 1,91 & 4,84 \\
\hline $\bar{x}$ & 34,79 & 35,16 & 35,61 & 37,48 & 37,98 & 38,87 & & & \\
\hline D.E & 1,92 & 2,89 & 1,16 & 2,82 & 2,01 & 2,01 & & & \\
\hline C.V & 5,52 & 8,20 & 3,27 & 7,53 & 5,30 & 5,17 & & & \\
\hline
\end{tabular}

espacial ni temporal en la condición trófica de la LLC durante todo el tiempo de muestreo. Esta ausencia de variación temporal también fue informada para el lago del Parque Norte de Medellín por Moreno Arbeláez \& Ramírez [9], donde los coeficientes de variación se informaron entre 8,2 y $13 \%$; de igual forma, Sierra [12] afirma que en los lagos ubicados en las zonas tropicales, las variaciones a lo largo de varios meses de muestreo es mínima, por su ubicación geográfica en la zona ecuatorial.

\section{5 ÍNDICE DE ESTADO TRÓFICO DE CARLSON MODIFICADO POR TOLEDO.}

Este índice permitió hacer un análisis cualitativo y cuantitativo de los parámetros analizados. Los resultados que se obtuvieron al aplicar las ecuaciones, se interpretaron según los criterios presentados en la tabla 4. El valor adimensional obtenido en cada punto, permitió clasificarlo en una categoría trófica. Los resultados encontrados para todos los puntos se resumen en la tabla 10.

En la revisión bibliográfica no se encontraron estudios en los cuales se haya aplicado el IETM a la LLC y, por tanto, no existen valores que permitan hacer una comparación de los resultados obtenidos.

Según el IETM, la LLC para la transparencia de la columna de agua y para la concentración de clorofila "a" se clasificó como ultraoligotrófica a oligotrófica, el comportamiento es similar 
Tabla 10. Estado trófico según el IETM en la laguna de La Cocha.

\begin{tabular}{|c|c|c|c|c|c|c|c|}
\hline \multirow{2}{*}{ Punto } & \multirow{2}{*}{ Estado trófico } & \multicolumn{6}{|c|}{ Mes de muestreo } \\
\hline & & Ene. & Feb. & Mar. & Abr. & May. & Jun. \\
\hline \multirow[t]{2}{*}{1} & IETM DS. & Ultra. & Oligo. & Ultra. & Ultra. & Oligo. & Oligo. \\
\hline & IETM Cl."a" & Oligo. & Oligo. & Oligo. & Oligo. & Oligo. & Oligo. \\
\hline \multirow[t]{2}{*}{2} & IETM DS. & Ultra. & Oligo. & Ultra. & Ultra. & Ultra. & Oligo. \\
\hline & IETM CI."a" & Ultra. & Oligo. & Oligo. & Oligo. & Oligo. & Oligo. \\
\hline \multirow[t]{2}{*}{3} & IETM DS. & Ultra. & Ultra. & Ultra. & Oligo. & Ultra. & Oligo. \\
\hline & IETM Cl."a" & Oligo. & Oligo. & Oligo. & Oligo. & Oligo. & Oligo. \\
\hline \multirow{2}{*}{4} & IETM DS. & Ultra. & Ultra. & Ultra. & Ultra. & Ultra. & Oligo. \\
\hline & IETM Cl."a" & Oligo. & Oligo. & Oligo. & Oligo. & Oligo. & Oligo. \\
\hline \multirow{2}{*}{5} & IETM DS. & Ultra. & Ultra. & Ultra. & Ultra. & Oligo. & Oligo. \\
\hline & IETM Cl."a" & Oligo. & Oligo. & Oligo. & Oligo. & Oligo. & Oligo. \\
\hline \multirow[t]{2}{*}{6} & IETM DS. & Ultra. & Ultra. & Ultra. & Oligo. & Oligo. & Oligo. \\
\hline & IETM Cl."a" & Oligo. & Oligo. & Oligo. & Oligo. & Oligo. & Oligo. \\
\hline \multirow[t]{2}{*}{7} & IETM DS. & Ultra. & Ultra. & Ultra. & Oligo. & Oligo. & Oligo. \\
\hline & IETM Cl."a" & Oligo. & Oligo. & Oligo. & Oligo. & Oligo. & Oligo. \\
\hline \multirow[t]{2}{*}{8} & IETM DS. & Ultra. & Ultra. & Ultra. & S.D & Oligo. & Oligo. \\
\hline & IETM Cl."a" & Oligo. & Oligo. & Oligo. & S.D & Oligo. & Oligo. \\
\hline \multirow[t]{2}{*}{9} & IETM DS. & Ultra. & Ultra. & Ultra. & Oligo. & Oligo. & Oligo. \\
\hline & IETM Cl."a" & Oligo. & Oligo. & Oligo. & Oligo. & Oligo. & Oligo. \\
\hline \multirow{2}{*}{10} & IETM DS. & Oligo. & Oligo. & Ultra. & Oligo. & Oligo. & Oligo. \\
\hline & IETM Cl."a" & Oligo. & Oligo. & Oligo. & Oligo. & Oligo. & Oligo. \\
\hline
\end{tabular}

Ultra. = ultraoligotrófico; Oligo. = oligotrófico; Meso. = mesotrófico y Eu. = Eutrófico S.D: sin datos; D.S: disco Sechhi; Cl. "a": clorofila "a"

para todos los puntos durante todos los meses de muestreo en los que se realizó está investigación. La clasificación trófica de la LLC como ultraoligotrófica a oligotrófica, evidencia una buena calidad del agua, y se espera encontrar bajas concentraciones de nutrientes y buena oxigenación.
Para observar con mayor claridad las variaciones entre los puntos y entre los meses de muestreo, se hizo un análisis estadístico con los valores del IETM promedio, cuyos resultados se observan en la tabla 11. 
Tabla 11. Valores promedios, X, D.E y C.V para el IETM en la Laguna de La Cocha.

\begin{tabular}{|c|c|c|c|c|c|c|c|c|c|}
\hline \multirow{2}{*}{ Puntos } & \multicolumn{6}{|c|}{ Valores promedios IETM } & \multirow{2}{*}{$\overline{\mathrm{x}}$} & \multirow{2}{*}{ D.E } & \multirow{2}{*}{$\begin{array}{l}\text { C.V } \\
\%\end{array}$} \\
\hline & Ene. & Feb. & Mar. & Abr. & May. & Jun. & & & \\
\hline 1 & 29,54 & 34,08 & 29,75 & 30,15 & 34,70 & 31,65 & 31,64 & 2,06 & 6,52 \\
\hline 2 & 26,77 & 34,64 & 29,41 & 30,37 & 30,90 & 31,75 & 30,64 & 2,38 & 7,76 \\
\hline 3 & 29,04 & 27,99 & 31,31 & 33,77 & 30,16 & 33,72 & 31,00 & 2,19 & 7,07 \\
\hline 4 & 29,79 & 27,41 & 29,31 & 31,24 & 30,97 & 33,64 & 30,39 & 1,92 & 6,30 \\
\hline 5 & 29,12 & 28,90 & 31,50 & 30,68 & 32,86 & 33,05 & 31,02 & 1,63 & 5,26 \\
\hline 6 & 29,67 & 28,23 & 31,15 & 32,09 & 33,64 & 34,24 & 31,50 & 2,10 & 6,68 \\
\hline 7 & 31,71 & 28,66 & 30,72 & 32,58 & 34,38 & 35,20 & 32,21 & 2,19 & 6,81 \\
\hline 8 & 29,70 & 29,04 & 32,24 & S.D & 34,27 & 35,88 & 32,23 & 2,61 & 8,09 \\
\hline 9 & 34,17 & 31,98 & 33,00 & 38,77 & 35,66 & 38,71 & 35,38 & 2,63 & 7,42 \\
\hline 10 & 32,43 & 35,32 & 32,06 & 36,79 & 36,59 & 36,06 & 34,87 & 1,92 & 5,50 \\
\hline $\bar{x}$ & 30,19 & 30,63 & 31,04 & 32,94 & 33,41 & 34,39 & & & \\
\hline D.E & 1,96 & 2,90 & 1,18 & 2,84 & 2,04 & 2,04 & & & \\
\hline C.V & 6,49 & 9,48 & 3,82 & 8,64 & 6,11 & 5,93 & & & \\
\hline
\end{tabular}

Los resultados de la media, aplicando el IETM, clasificaron a la LLC en las categorías ultraoligotrófica y oligotrófica. El valor de la media obtenida se utilizó para calcular la desviación estándar, encontrándose que la concentración de datos alrededor de la media es alta, por lo que se deduce que existe homogeneidad y no se encuentran valores extremos, pues la variación es pequeña $(<3)$.

Posteriormente, se calculó el coeficiente de variación para cada punto de muestreo y para cada mes de muestreo; este coeficiente arrojó valores inferiores a $10 \%$, lo que permite afirmar que, según las orientaciones de Canavos [39], los datos fueron homogéneos tanto en los meses de mayor como de menor precipitación. En el punto 5, ubicado en el centro de la LLC, se encontró el menor valor del coeficiente variación, con un $5,26 \%$, y el valor más alto fue de $8,09 \%$ en el punto 8 , ubicado en la vereda Carrizo. En el mes de marzo se encontró el valor más bajo del coeficiente de variación, $3,82 \%$, y en el mes de febrero se encontró el valor más alto con un $9,48 \%$.

Los valores del coeficiente de variación para el IETM promedio, indicaron que la condición trófica de la LLC durante todo el tiempo de muestreo es similar en los 10 puntos de muestreo y en todos los meses de muestreo, la LLC tendió a permanecer en una condición 
ultra a oligotrófica. Esta ausencia de variación temporal fue informada también por De Lucena et al. [40] en Brasil, en los cuerpos de agua ubicados en la cuenca hidrográfica del río Taperoá, cuyo coeficiente de variación del IETM fue de $9,4 \%$, y para el lago del Parque Norte de Medellín [9], donde los coeficientes de variación se informaron con valores de 8,2 \%.

\section{CONCLUSIONES}

La cuantificación de la clorofila "a", a pesar de sus limitaciones, es un método económico y rápido, siendo extremadamente útil en la determinación de la calidad del agua. De igual manera es un parámetro que puede servir en estudios del estado fisiológico del fitoplacton.

El estado trófico, según los índices de OCDE, IET e IETM, indicó que no existe variación espacial, ni temporal para la cantidad de biomasa y la transparencia en la LLC, lo que evidencia la multidimensionalidad del concepto de calidad de agua y la imposibilidad de establecer una limitación estricta entre las diferentes categorías tróficas.

Según los índices de estado trófico IET e IETM, la LLC se clasificó como oligotrófica y ultraoligotrófica. Esta clasificación es típica de lagos de alta montaña con bajos procesos de contaminación de origen aloctóno y autóctono, que evidencian una buena calidad de agua con poca proliferación de algas y alta oxigenación.

Para hacer un seguimiento constante a la calidad del agua de la LLC, y teniendo en cuenta su importancia como humedal Ramsar, es necesario implementar un plan de monitoreo constante que incluya parámetros físicos, químicos y biológicos para poder observar las tendencias en el tiempo.

\section{AGRADECIMIENTOS}

Las autoras agradecen al Grupo de Investigación Biotics de la Universidad Nacional Abierta y a Distancia UNAD y al Grupo de Investigación Ambiental GIA de la Universidad Mariana, quienes financiaron la investigación.

\section{BIBLIOGRAFÍA}

[1] Margalef, R. (1983). Limnología. Barcelona: Ediciones Omega.

[2] Gunkel, G. (2003). Limnología de un lago tropical de alta montaña, en Ecuador: características de los sedimentos y tasa de sedimentación. En: Revista de Biología Tropical, 51(2), pp. 381-392.

[3] Alcaldía Municipal de Pasto. (2012). Plan de ordenamiento territorial de Pasto. Cuaderno dimensión sociocultural. San Juan de Pasto, p. 244.

[4] Fondo Mundial para la Conservación de la Naturaleza. (2004). El agua dulce y la reducción de la pobreza: sirviendo a la gente, salvando la Naturaleza. WWF Internacional, p. 36.

[5] Ministerio del Medio Ambiente. Corponariño. (2011). Plan de manejo integral Humedal Ramsar laguna de la Cocha. San Juan de Pasto: CTPRINT Impresores.

[6] Fia, R., Texeira, A., Carteri, P., Pereira, 0. (2009). Estado trófico da água na bacia hidrográfica da Lagoa Mirim , RS , Brasil Trophic state of water in the watershed of Lake Mirim , RS , Brazil. Ambiente \& 
Água - An Interdisciplinary. En: Journal of Applied Science, (12), 132-141.

[7] Smith R., Smith, T. (2001). Ecología. Madrid: Pearson Educación.

[8] Condé, M. (2004). Grau de trofia em corpos d'água do Estado de São Paulo: Avaliacão dos métodos de monitoramento (tesis doctoral). Universidade de São Paulo. São Paulo. 238 p.

[9] Moreno Arbeláez, D., Ramírez, J. J. (2010). Variación temporal de la condición trófica del lago Parque Norte, Medellín (Antioquia), Colombia. En: Actualidades Biológicas, 32(92), 75-87.

[10] Nogueira, N., Ramírez, J. J. (1998). Variacao mensal da condicao trófica do Lago das Garcas (Sao Paulo, SP, Brasil). En: Acta Limnologica Brasiliensia, 10, 21-34.

[11] Chapra, S. (1997). Surface Water - Quality Modeling. New York: McGraw-Hill.

[12] Sierra, C. (2011). Calidad del agua. Evaluación y diagnóstico. Medellín: Universidad de Medellín.

[13] Ministerio del Medio Ambiente. SIGAM. (2004). Agenda Ambiental del municipio de Pasto. San Juan de Pasto.

[14] Bonilla M., Guillot, G. (2003). Prácticas de Ecología. Santa Fe de Bogotá: Editorial Universidad Nacional de Colombia.

[15] APHA, A. \& W. (2012). Standard Methods for the Examination of Water and Wastewater. American Public Health Association, American Water Works Association \& Water Environment
Federation. (E. W. Rice, R. B. Baird, A. D. Eaton, \& L. S. Clesceri, Ed.) (22nd ed.). Washington.

[16] Calazans, M., Ovruski, B., De Souza, H., Konig, A. (1997). Utilização dos índices do estado trófico (IET) e de qualidade da água (IQA) na caracterização limnológica e sanitária das Lagoas de Bonfim, Extremóz e Jiqui (RN) - Análise preliminar. En http://www.bvsde.paho.org/bvsacd/ abes97/trofico.pdf (febrero 25 de 2014).

[17] Martino, P. (1989). Curso básico sobre eutrofización. En: http://www.bvsde. paho.org/bvsacd/scan/020465.pdf (abril 20 de 2014).

[18] Salas, H. \& Martino, P. (2001). Metodologías simplificadas para la evaluación de eutrofia en lagos cálidos tropicales. (C. P. de I. S. y A. Cepis, Ed.) Centro Panamericano de Ingeniería Sanitaria y Ambiental. Cepis. En: http:// www.bvsde.ops-oms.org/bvsaca/e/ fulltext/eutrof/eutrof.pdf (abril 20 de 2014).

[19] Ludovisi, A., Poletti, A. (2003). Use of thermodynamic indices as ecological indicators of the development state of lake ecosystems. 1. Entropy production indices. En: Ecological Modelling, 159(23), 203-222.

[20] Carlson, R. (1977). A trophic state index for lakes. En: Limnology and Oceanography, 22(2), 361-369.

[21] Correa, G., Cunha, H. (2010). Avaliacao do grau de eutrofizacao de dos igarapés urbanos de Manaus- AM. In Anais da XIX jornada de iniciacao cientifica do INPA_ 
PIBIC/CNPq - PAIC/FAPEAM (p. 4). En: http://pibic.inpa.gov.br/clima.html (julio 5 de 2014).

[22] Moreno Franco, D., Quintero, J., López, A. (2010). Métodos para identificar, diagnosticar y evaluar el grado de eutrofia. En: Revista Contactos, 25-33.

[23] Pena, L., Ferreira, C., Almeida, M. (2004). Comportamento dos índices do estado trófico de Carlson (IET) emodificado (IETM) no reservatório da UHE Luís Eduardo Magalhaes. Tocantins-Brasil. In Congreso Interamericano de Ingeniería Sanitaria y Ambiental (p. 6). En: http://www.bvsde. paho.org/bvsAIDIS/PuertoRico29/tocan. pdf (Abril 7 de 2014).

[24] Roldán, G., Ramírez, J. J. (2008). Limnología neotropical. Medellín: Universidad de Antioquia.

[25] López, J., Salas, J., Gómez, Y., Gómez, A., López, H., Ortega, A., Guerrero, C. (2008). Diagnóstico del estado de trofización y estudio de la variabilidad de los parámetros limnológicos y su interrelación con la producción de trucha arco iris en jaulas flotante en el lago Guamuez. San Juan de Pasto.

[26] Casallas, J., Gunkel, G. (2001). Algunos aspectos limnológicos de un lago altoandino: el lago San Pablo. En: Limnética, 215-232.

[27] Iltis, A., Carmouze, J., Lemoalle, J. (1991). Características físicoquímicas del agua. In C. Dejoux \& A. Iltis (Eds.). El lago Titicaca, síntesis del conocimiento limnológico actual. La Paz, Bolivia, pp. 107-114.
[28] Morales, G. (2004). Crecimiento y eficiencia alimentaria de trucha arco iris (Oncorhynchus mykiss) en jaulas flotantes bajo diferentes regímenes de alimentación (trabajo de grado). Universidad de Buenos Aires. 51 p.

[29] Díaz, M., Pedrozo, F., Reynolds, C., Temporetti, P. (2007). Chemical composition and the nitrogen-regulated trophic state of Patagonian lakes. En: Limnológica, 37(1), 17-27.

[30] Gunkel, G. (2000). Limnology of an Equatorial High Mountain Lake in Ecuador, lago San Pablo. En: Limnológica, 30, 113-120.

[31] Aubriot, L., Conde, D., Bonilla, S., Hein, V., Britos, A. (2005). Vulnerabilidad de una laguna costera en una reserva de biosfera: indicios recientes de eutrofización. En: Vila y Pizarro (Ed.). Taller internacional de eutrofización de lagos y embalses. En: CYTED XVIIB. Chile, pp. 65-85.

[32] Coelho, S., Gamito, S., Pérez-Ruzafa, A. (2007). Trophic state of Foz de Almargem coastal lagoon (Algarve, South Portugal) based on the water quality and the phytoplankton community. En: Estuarine, Coastal and Shelf Science, 71(1-2), 218231.

[33] Fontúrbel, F. (2005). Indicadores fisicoquímicos y biológicos del proceso de eutrofización del lago Titicaca (Bolivia). Ecología Aplicada, 4.

[34] Fraile, H., Pozo, E., Ciencias, F., Vasco, P. (1995). Evaluación del estado trófico y comparación de modelos relativos al fósforo en los embalses de Cerdanilla 
y Valparaíso (río Tera, Zamora). En: Limnética, 11(2), 29-37.

[35] Quirós, R. (2000). La eutrofización de las aguas continentales de Argentina. El agua en Iberoamérica: acuíferos lagos y embalses. CYTED. Subprograma XVII. Aprovechamientos y gestión de recursos hídricos. En: http://www.agro. uba.ar/users/quiros/Eutrofizacion/ EutroArgentina.pdf

[36] Velásquez, J., Jiménez, G., Sepúlveda, M. (2007). Determinación de la calidad ambiental de la Ciénaga Colombia. Caucasia-Antioquia-Colombia. En: Gestión y Ambiente, 10(4), 187-199.

[37] Betancourt, C., Suárez, R., Toledo, L. (2010). Variabilidadiónicay características tróficas del embalse Abreus, Cuba. En: Limnética, 29(2), 341-352.

[38] Li, Y., Liu, H., Zheng, N., Cao, X. (2010). Analysis of Trophic Status and its Influence Factors of Different Water Body Types in Xixi National Wetland Park, China. En: Procedia Environmental Sciences, 2, 768-780.

[39] Canavos, G. (1998). Probabilidad y estadística. Aplicaciones y métodos. México: MacGraw-Hill.

[40] De Lucena, J., Souza, R., Piava, L. \& Regina, C. (2006). Diagnóstico do estado trófico e aspectos limnológicos de sistemas aquáticos da Bacia Hidrográfica do Rio Taperoá, Trópico semi-árido Brasileiro. En: Revista de Biología E Ciencias Da Terra, 81-89. 